\title{
Metformin and Prostate Cancer: a New Role for an Old Drug
}

\author{
Jessica Whitburn ${ }^{1}$ - Claire M. Edwards ${ }^{1}$ - Prasanna Sooriakumaran ${ }^{1,2}$
}

Published online: 25 April 2017

(C) The Author(s) 2017. This article is an open access publication

\begin{abstract}
Purpose of Review Since epidemiological studies first demonstrated a potential positive effect of metformin in reducing cancer incidence and mortality, there has been an increased interest in not only better understanding metformin's mechanisms of action but also in exploring its potential anti-cancer effects. In this review, we aim to summarise the current evidence exploring a role for metformin in prostate cancer therapy.

Recent Findings Preclinical studies have demonstrated a number of antineoplastic biological effects via a range of molecular mechanisms. Data from retrospective epidemiological studies in prostate cancer has been mixed; however, there are several clinical trials currently underway evaluating metformin's role as an anti-cancer agent. Early studies have shown benefits of metformin to inhibit cancer cell proliferation and improve metabolic syndrome in prostate cancer patients receiving androgen deprivation therapy (ADT).

Summary While the body of evidence to support a role for metformin in prostate cancer therapy is rapidly growing, there is still insufficient data from randomised trials, which are currently still ongoing. However, evidence so far suggests
\end{abstract}

This article is part of the Topical Collection on Urosurgery

Jessica Whitburn

jesswhitburn@gmail.com; jessica.whitburn@balliol.ox.ac.uk

1 Nuffield Department of Surgical Sciences, University of Oxford, Botnar Research Centre Old Road, Oxford OX3 7LD, UK

2 Department of Urology, University College London Hospitals NHS Foundation Trust, London, UK metformin could be a useful adjuvant agent, particularly in patients on ADT.

Keywords Metformin $\cdot$ Biguanide $\cdot$ Cancer $\cdot$ Prostate $\cdot$ AMPK

\section{Introduction}

Prostate cancer (PCa) remains the most commonly diagnosed male cancer in the Western world and is the second leading cause of cancer-related deaths in men [1]. Over the last 10 years, increasing evidence is demonstrating that biguanide drugs such as metformin may be beneficial in the prevention and treatment of a range of cancers, including prostate cancer [2]. Metformin is the most commonly used oral anti-diabetic drug in the world. It is also used in the treatment of polycystic ovary syndrome, and is being investigated as a potential antiviral agent. It has been in clinical use for more than 50 years and has a good safety record with limited toxicity. Lower cancer incidence and cancer-specific deaths have been reported among diabetics on metformin compared to diabetics on other anti-diabetic medications $[3 \cdot, 4]$. These results are in keeping with in vitro work, which has shown metformin to be anti-proliferative to a range of cancer cell lines, and in vivo work where metformin has been shown to inhibit the growth of cancer xenografts [5, 6]. Higher insulin and c-peptide levels have been associated with poorer outcomes in cancer patients $[7,8]$, and metformin has been shown to be effective at reducing insulin levels, even in non-diabetic patients [9]. Given its excellent safety profile, low cost, and minimal side effects, it is an attractive candidate as a potential cancer therapeutic. However, we still have limited knowledge of the specific molecular mechanisms underlying its beneficial properties. 


\section{Metformin: Mechanism of Action}

Metformin was originally derived from Galega officinalis (French lilac), a plant reputedly used to treat diabetes-like conditions in medieval Europe [10]. Despite its widespread use in the treatment of type 2 diabetes, details of the mechanism of action of metformin in this disease were only recently elucidated, and gaps in knowledge remain [11]. It is thought to exert its anti-cancer effect via two mechanisms - directly by acting on the tumour and indirectly by lowering systemic insulin levels (Fig. 1). The direct (insulin-independent) effect is via inhibition of the mitochondrial electron transport chain (ETC) and consequent activation of the enzyme adenosine mono-phosphate-activated protein kinase (AMPK) [12]. AMPK is an energy sensing/signalling protein that is a central control point for maintaining energy homeostasis. It detects an increase in the ratio of AMP to ATP secondary to cellular stresses (e.g. glucose deprivation, hypoxia, and oxidative stress). When activated, it increases cellular ATP by inhibiting anabolic pathways and stimulating catabolic pathways to produce ATP $[13,14]$. Activation of AMPK leads to downstream inhibition of mammalian target of rapamycin complex-1 (mTORC1) signalling, and activation of the tumour suppressor tuberous sclerosis complex 2 (TSC2, tuberin) [15]. mTOR is a key mediator of the phosphatidylinositol-3-kinase/protein kinase $\mathrm{B} / \mathrm{Akt}(\mathrm{PI} 3 \mathrm{~K} / \mathrm{PKB} / \mathrm{Akt}$ ) signalling pathway, which is one of the most frequently deregulated pathways in human cancer [16]. Inhibition of mTOR leads to attenuation of protein synthesis and tumour cell growth and proliferation through downstream targets, 4E-BP1 and p70S6K1 [17-20]. Additionally, inhibition of the Krebs cycle may have further direct effects on other metabolic pathways such as lipid synthesis and beta-oxidation that are known to be important in prostate cancer metabolism [21]. Fatty acid synthase (FAS), a key enzyme of lipogenesis, is known to be upregulated in cancer to allow high rates of de novo fatty acid production. AICAR, an activator of AMPK, has been shown to inhibit FAS in prostate cancer cells [22] which may further contribute to metabolic stress.

The indirect (insulin-dependent) actions of metformin occur via inhibition of hepatic gluconeogenesis. Activation of AMPK in the liver leads to inhibition of the transcription of key gluconeogenesis genes, and stimulates glucose uptake in muscle, thus reducing fasting blood glucose and insulin levels [10, 23]. Insulin is known to have mitogenic and pro-survival effects, with tumour cells often expressing high levels of the insulin receptor. High insulin levels are known to be an adverse prognostic factor for a number of cancers, including breast, colon, and prostate cancer [8, 24, 25], and metformin has been shown to be able to lower systemic insulin levels, even in nondiabetic patients [9]. Reduced circulating insulin leads to a subsequent down-regulation of the phosphoinositide-3-kinase (PI3K) axis. The PI3K pathway is involved in growth, proliferation, differentiation, and motility, and following the androgen receptor (AR) pathway is the second major driver of prostate cancer growth. The indirect actions are systemic and therefore do not require metformin to be able to accumulate within the tumour which maybe important for poorly vascularised tumours.

Which mechanism predominates in prostate cancer is unknown but it is likely that both pathways provide anti-cancer benefits. Also, it still remains unclear whether AMPK activation is essential for metformin activity, as its ability to inhibit mTORC1 has also been demonstrated via AMPKindependent pathways [26]. Further research is ongoing to better clarify the mechanisms that predominate in the cancer setting.

\section{Epidemiological Evidence}

Metformin's inhibitory effects on various pro-oncogenic pathways prompted epidemiologist to retrospectively analyse the effect of metformin treatment on type 2 diabetic patients with cancer. Evans and colleagues were the first to show that taking metformin may be associated with reduced risk of cancer in patients with type 2 diabetes, and that the longer the period of exposure the greater the benefit [3•]. A more recent study in the same geographical setting showed that in a cohort of 8000 patients with type 2 diabetes, cancer was diagnosed in $7.3 \%$ of metformin users compared with $11.6 \%$ of non-users, and that higher doses of metformin were associated with the greatest reduction in cancer risk [27]. Further studies have compared metformin therapy to other anti-diabetic therapies and found that while diabetics had an increased cancer mortality compared to non-diabetics, those on metformin had a lower cancer mortality compared to those taking other anti-diabetic medications such as sulfonylureas or insulin $[4,28]$. The effects of metformin have also been analysed in organ-specific disease. In prostate cancer, several studies have found a beneficial effect of metformin in reducing prostate cancer incidence and improving overall survival [29-33]. A recent large population-based study found that metformin users were approximately $16 \%$ less likely to be diagnosed with prostate cancer than non-users, with an inverse relationship between prostate cancer risk and duration, intensity of use, and cumulative dose [34•]. Despite the size of these epidemiological studies, they are all retrospective examining only diabetic patients, and there is considerable confounding and heterogeneity between the studies. To date, only three randomised trials examining metformin's effect on cancer survival have been published, all in the late disease setting. These have found no difference in overall survival in patients treated with metformin in combination with standard systemic therapies [35-37]. However, a recent study by Higurashi et al. [38] examined metformin's role in chemoprevention of colorectal 


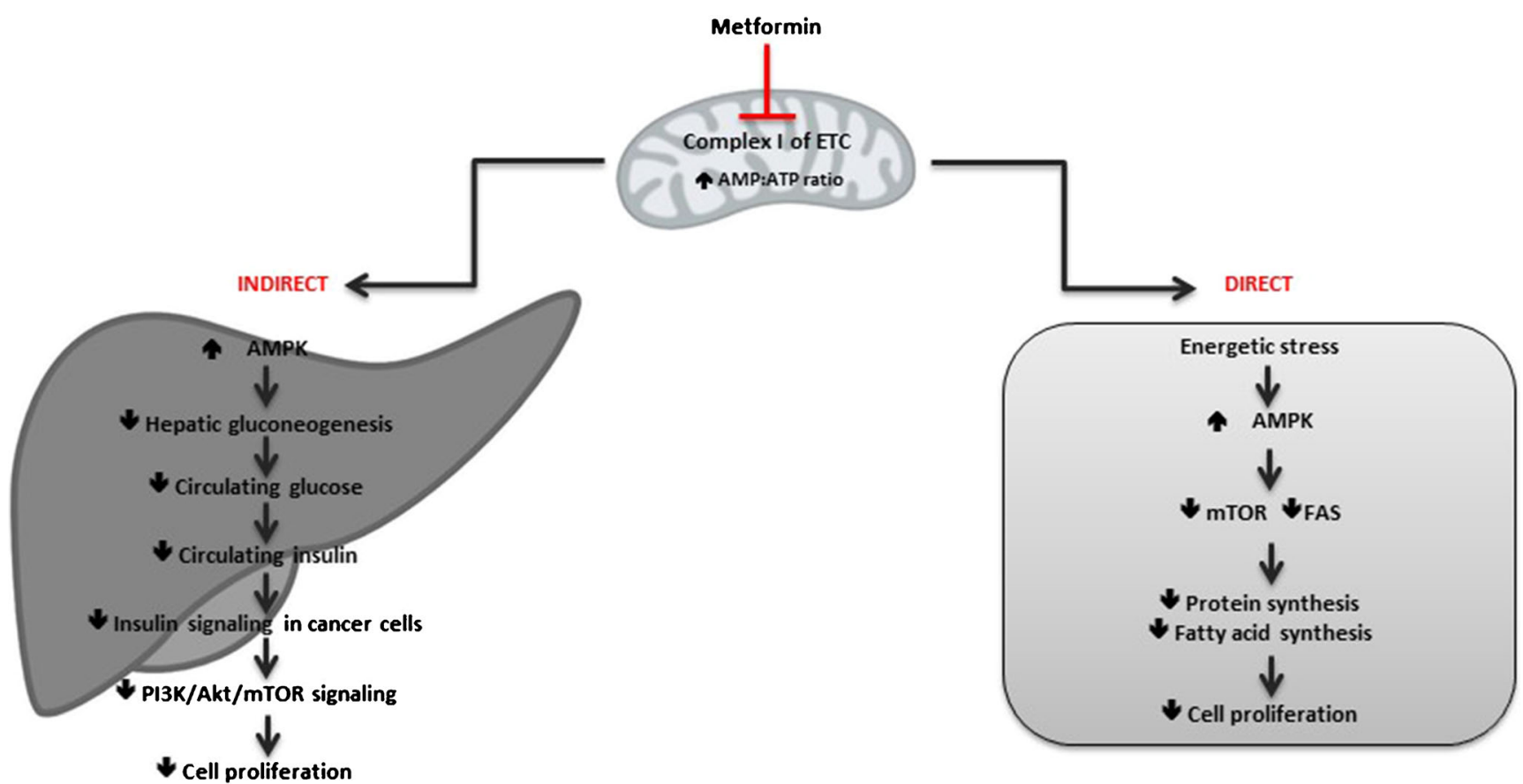

Fig. 1 Overview of the direct and indirect anti-cancer effect of metformin

cancer and found that low-dose metformin for 1 year reduced the prevalence and number of metachronous colorectal adenomas or polyps in patients with high risk of adenoma recurrence after polypectomy, with no serious adverse events during the trial. In view of this, many questions still remain with regards to metformin's role in oncology therapy. Will metformin be an effective anti-cancer agent in non-diabetic patients? Is the anti-diabetic dose the best anti-cancer dose? Will metformin only be effective in certain subgroups of patients, or in certain malignancies? Will metformin's effects be limited to cancer prevention or does it have a role in cancer treatment? There are currently several randomised clinical trials being undertaken to answer these questions both in prostate cancer and other malignancies, and the results of these are eagerly awaited.

\section{Role in Preventing Metabolic Syndrome}

Alongside its potential anti-cancer role, clinical trials have also examined a role for metformin in preventing androgen deprivation therapy (ADT)-induced metabolic syndrome in prostate cancer. Whilst ADT is the mainstay of treatment for men with advanced prostate cancer, improving patient's survival, it can also cause significant morbidity and a decline in quality of life (QOL). Body composition changes, insulin resistance, sexual dysfunction, fatigue, reduced bone mineral density (BMD), hyperlipidaemia, metabolic syndrome, and acute coronary syndrome are all reported adverse effects of ADT as a consequence of reduced levels of circulating testosterone and raised insulin levels [39, 40]. Men with prostate cancer have higher rates of non-cancer mortality and cardiovascular morbidity, and some of this excess risk can be attributed to androgen deprivation therapy [41]. Studies have shown that even short-term use of ADT significantly increases fat mass and decreases insulin sensitivity in men with prostate cancer [42]. Metabolic syndrome is not only associated with cardiovascular disease (CVD) but may also have an adverse effect on prostate cancer prognosis. Insulin has been shown to promote local androgen synthesis by prostate cancer cells, which is thought to represent a resistance mechanism to castration [43]. A retrospective study showed the presence of metabolic syndrome was associated with a shorter median time to progression and shorter median overall survival in prostate cancer patients receiving ADT [44]. If metformin is able to lower the hyperinsulinaemia and improve the metabolic syndrome seen, then there will be a strong rationale to examine the benefits of combining metformin with ADT, with a potential role for metformin in improving both the tolerability and efficacy of ADT. There is currently only one randomised trial reporting on this which found that a combination of metformin with a low glycaemic index-diet and exercise programme improved the abdominal girth, weight, BMI, and systolic blood pressure of men with prostate cancer beginning ADT; however, no difference was noted in the biochemical markers of insulin resistance $[45 \bullet \cdot]$. Further trials are needed to determine if metformin has a role in reducing ADTinduced metabolic syndrome, and if this corresponds in a reduction in CVD-related deaths, prostate cancer-specific death, and all-cause mortality. 


\section{Biological Effects}

Metformin has been shown to inhibit the proliferation of a range cancers, including breast, endometrial, ovarian, colon, and prostate $[5,18,46-50]$. Its effect can be seen in both in vitro cell lines and in mouse tumour models. Interestingly, Ben Sahara et al. [5] found that while metformin was antiproliferative to prostate cancer cells, normal epithelial prostate cells were barely affected by metformin suggesting a cancerspecific effect. However, it is important to note that many of these experiments have used exceedingly high doses, up to 100 to 300 times the conventional anti-diabetic dose. More recently, there have been several 'window-of-opportunity' biomarker trials of metformin in cancer patient's pre-surgery examining immunohistochemical markers, typically using anti-diabetic therapeutic doses. Metformin has been shown to reduce expression of Ki67, a marker of cell proliferation in breast, endometrial, and prostate cancers [51-53, 54••, 55]. Joshua et al. matched biopsy and prostatectomy samples in 24 non-diabetic patients given a short (median duration 41 days) course of metformin prior to prostatectomy. They found metformin was well tolerated and significantly reduced the Ki67 index by almost $30 \%$ per patient. There was also a nonsignificant trend towards PSA reduction in these patients. Weaknesses of this study include the fact that it was a small pilot trial $(n=22)$, not placebo controlled, and that the tissue examined was obtained by different methods (biopsy vs prostatectomy); however, it provides strong rationale for further studies to not only examine disease and survival end-points but to also incorporate tissue and serum markers to better elucidate metformin's actions.

Interestingly, metformin's biological effects are not limited to inhibiting cancer proliferation, but include effects on epithelial to mesenchymal transition (EMT), bone turnover, the androgen receptor, and cancer stem cells. EMT is an important step in prostate cancer invasion and metastases, conferring upon cancer cells the ability to invade basement membranes and metastasize to distant sites. EMT can be characterised by expression of certain markers by the cell. Epithelial markers include E-cadherin, cytokeratin, and desmoplakin, while mesenchymal markers include vimentin, slug, snail, and twist. Expressions of twist and vimentin have been shown to be predictive of biochemical recurrence in prostate cancer patients after radical prostatectomy for localised disease [56]. In melanoma and breast cancer cells, metformin is able to inhibit expression of markers of EMT via activating AMPK $[57,58]$. It has also been shown to reverse the mesenchymal phenotype of prostate cancer cells in vitro and inhibit their invasive phenotype [59].

Prostate cancer most commonly metastasises to the bone leading to skeletal-related events. In combination with this, ADT leads to loss of bone mineral density and increased risk of fracture. Metformin has been shown to enhance osteoblast and inhibit osteoclast differentiation in vitro [60,61], and to prevent bone loss in ovariectomized rats [62], raising the possibility that metformin may also be protective against cancerinduced bone disease in these high-risk patients.

The androgen receptor (AR) plays a vital role in prostate cancer, regulating a multitude of events including proliferation, apoptosis, migration, invasion, and differentiation. ADT remains the standard treatment for advanced prostate cancer; however, almost all patients treated with ADT will eventually relapse into castration-resistant prostate cancer (CRPC). Studies have demonstrated that persistent AR signalling remains the key driver in the progression to CRPC, and AR down-regulation is considered a preventive strategy for prostate cancer [63]. Metformin has been shown to reduce AR protein levels in a dose-dependent manner in androgen receptor-positive cell lines [49], and repress the AR signalling pathway via down-regulation of AR mRNA [64], again supporting a potential role for metformin in combination with ADT therapy.

Cancer stem cells (CSCs) are a subset of tumour cells that are resistant to many anti-cancer treatments, and are able to self-renew and regenerate the various cell types that make up a tumour [65]. Prostate CSCs are known to be resistant to most conventional cancer therapies and are thought to contribute to local invasion and bone metastasis [66] as well as the development of CRPC [67]. Metformin has been shown to effectively target breast and pancreatic CSCs, and it has also been shown to enhance the effectiveness of standard therapies [68, 69].

Iliopoulos et al. found metformin in combination with the chemotherapeutic drug doxorubicin was more effective than either drug alone at blocking breast, prostate, and lung cancer growth and preventing relapse in mouse xenograft models. Interestingly, metformin had a comparable effect on tumour regression and preventing relapse when combined with a fourfold decreased dose of doxorubicin that was not effective as a monotherapy, suggesting a complementary role for metformin, potentially allowing lower doses of toxic drugs to be used, improving their tolerability and side effects [70]. Indeed, there have been a number of studies showing synergistic or enhanced effects of metformin when used in combination with a range of other therapies including commonly used medications such as statins and aspirin [71-73]. The combination of metformin with ADT has been shown to enhance the reduction of prostate tumour growth in mouse models [74], and metformin has been shown to increase the sensitivity of prostate cancer cells to radiotherapy treatment $[75,76]$. This provides a strong rationale for the addition of metformin to current clinical trial treatment regimens, and indeed, metformin has been added as an arm to the STAMPEDE trial (Systematic Therapy for Advancing or Metastatic PCa), a UK trial which has recruited greater than 4000 patients to date. 


\section{Conclusions}

Metformin is an attractive anti-cancer agent in view of its well-documented safety record, well-characterised pharmacodynamics profile, and low cost as a generic drug. Over the last 10 years, interest in this field has grown exponentially with a combination of epidemiological, clinical, and preclinical data supporting a potential anti-cancer role for metformin. However, many questions still remain. It is still unclear if metformin will be effective in a non-diabetic population, and if its effects are limited to particular patient populations (e.g. specific malignancies or cancer cells with specific metabolic pathway mutations). Alongside this, much of the preclinical work has used supra-physiological doses of metformin. There are currently a large number of studies worldwide being undertaken examining metformin in a range of cancers; however, many of these are using metformin at the conventional antidiabetic doses. It awaits to be seen if these doses will be effective or if further phase I and phase II clinical trials will be required to assess metformin's tolerability and efficacy as an anti-cancer agent at higher doses. Well-designed prospective controlled clinical trial outcomes will be important to provide more definitive answers regarding the efficacy of metformin in prevention and treatment of prostate cancer, and its role as a potential adjuvant therapy. However, data so far is very compelling for metformin's role in the treatment of prostate cancer, particularly in view of its potential synergy with currently used treatments such as ADT and radiotherapy.

\section{Compliance with Ethical Standards}

Conflict of Interest Jessica Whitburn and Claire M. Edwards each declare no potential conflicts of interest.

Prasanna Sooriakumaran is a section editor for Current Urology Reports.

Human and Animal Rights and Informed Consent This article does not contain any studies with human or animal subjects performed by any of the authors.

Open Access This article is distributed under the terms of the Creative Commons Attribution 4.0 International License (http:// creativecommons.org/licenses/by/4.0/), which permits unrestricted use, distribution, and reproduction in any medium, provided you give appropriate credit to the original author(s) and the source, provide a link to the Creative Commons license, and indicate if changes were made.

\section{References}

Papers of particular interest, published recently, have been highlighted as:

- Of importance,

• Of major importance

1. Siegel RL, Miller KD, Jemal A. Cancer statistics, 2015. CA Cancer J Clin. 2015;65(1):5-29.
2. Gronich N, Rennert G. Beyond aspirin-cancer prevention with statins, metformin and bisphosphonates. Nat Rev Clin Oncol. 2013;10(11):625-42.

3. Evans JM, et al. Metformin and reduced risk of cancer in diabetic patients. BMJ. 2005;330(7503):1304-5. First epidemiological paper to link metformin use and reduced cancer risk

4. Bowker SL, et al. Increased cancer-related mortality for patients with type 2 diabetes who use sulfonylureas or insulin. Diabetes Care. 2006;29(2):254-8.

5. Ben Sahra I, et al. The antidiabetic drug metformin exerts an antitumoral effect in vitro and in vivo through a decrease of cyclin D1 level. Oncogene. 2008;27(25):3576-86.

6. Vazquez-Martin A, Oliveras-Ferraros C, Menendez JA. The antidiabetic drug metformin suppresses HER2 (erbB-2) oncoprotein overexpression via inhibition of the mTOR effector p70S6K1 in human breast carcinoma cells. Cell Cycle. 2009;8(1):88-96.

7. Lehrer S, et al. Serum insulin level, disease stage, prostate specific antigen (PSA) and Gleason score in prostate cancer. Br J Cancer. 2002;87(7):726-8.

8. Ma J, et al. Prediagnostic body-mass index, plasma C-peptide concentration, and prostate cancer-specific mortality in men with prostate cancer: a long-term survival analysis. Lancet Oncol. 2008;9(11):1039-47.

9. Goodwin PJ, et al. Insulin-lowering effects of metformin in women with early breast cancer. Clin Breast Cancer. 2008;8(6):501-5.

10. Hardie DG, Ross FA, Hawley SA. AMPK: a nutrient and energy sensor that maintains energy homeostasis. Nat Rev Mol Cell Biol. 2012;13(4):251-62.

11. Pollak MN. Investigating metformin for cancer prevention and treatment: the end of the beginning. Cancer Discov. 2012;2(9): 778-90.

12. Owen MR, Doran E, Halestrap AP. Evidence that metformin exerts its anti-diabetic effects through inhibition of complex 1 of the mitochondrial respiratory chain. Biochem J. 2000;348(Pt 3):607-14.

13. Merrill GF, et al. AICA riboside increases AMP-activated protein kinase, fatty acid oxidation, and glucose uptake in rat muscle. Am J Phys. 1997;273(6 Pt 1):E1107-12.

14. Hardie DG, Carling D, Carlson M. The AMP-activated/SNF1 protein kinase subfamily: metabolic sensors of the eukaryotic cell? Annu Rev Biochem. 1998;67:821-55.

15. Inoki K, Zhu T, Guan KL. TSC2 mediates cellular energy response to control cell growth and survival. Cell. 2003;115(5):577-90.

16. Liu P, et al. Targeting the phosphoinositide 3-kinase pathway in cancer. Nat Rev Drug Discov. 2009;8(8):627-44.

17. Dowling RJ, et al. Metformin inhibits mammalian target of rapamycin-dependent translation initiation in breast cancer cells. Cancer Res. 2007;67(22):10804-12.

18. Zakikhani M, et al. Metformin is an AMP kinase-dependent growth inhibitor for breast cancer cells. Cancer Res. 2006;66(21):1026973.

19. Mahalingam D, et al. Targeting the mTOR pathway using deforolimus in cancer therapy. Future Oncol. 2009;5(3):291-303.

20. Kalender A, et al. Metformin, independent of AMPK, inhibits mTORC1 in a rag GTPase-dependent manner. Cell Metab. 2010;11(5):390-401.

21. Zadra G, Photopoulos C, Loda M. The fat side of prostate cancer. Biochim Biophys Acta. 2013;1831(10):1518-32.

22. Xiang $X$, et al. AMP-activated protein kinase activators can inhibit the growth of prostate cancer cells by multiple mechanisms. Biochem Biophys Res Commun. 2004;321(1):161-7.

23. Shaw RJ, et al. The kinase LKB1 mediates glucose homeostasis in liver and therapeutic effects of metformin. Science. 2005;310(5754):1642-6.

24. Goodwin PJ, et al. Insulin- and obesity-related variables in earlystage breast cancer: correlations and time course of prognostic associations. J Clin Oncol. 2012;30(2):164-71. 
25. Wolpin BM, et al. Insulin, the insulin-like growth factor axis, and mortality in patients with nonmetastatic colorectal cancer. J Clin Oncol. 2009;27(2):176-85.

26. Ben Sahra I, et al. Metformin in cancer therapy: a new perspective for an old antidiabetic drug? Mol Cancer Ther. 2010;9(5):1092-9.

27. Libby G, et al. New users of metformin are at low risk of incident cancer: a cohort study among people with type 2 diabetes. Diabetes Care. 2009;32(9):1620-5.

28. Currie CJ, et al. Mortality after incident cancer in people with and without type 2 diabetes: impact of metformin on survival. Diabetes Care. 2012;35(2):299-304.

29. Ruiter R, et al. Lower risk of cancer in patients on metformin in comparison with those on sulfonylurea derivatives: results from a large population-based follow-up study. Diabetes Care. 2012;35(1): 119-24.

30. Murtola TJ, et al. Antidiabetic medication and prostate cancer risk: a population-based case-control study. Am J Epidemiol. 2008;168(8):925-31.

31. He XX, et al. Thiazolidinediones and metformin associated with improved survival of diabetic prostate cancer patients. Ann Oncol. 2011;22(12):2640-5.

32. Margel D, et al. Metformin use and all-cause and prostate cancerspecific mortality among men with diabetes. J Clin Oncol. 2013;31(25):3069-75.

33. Wright JL, Stanford JL. Metformin use and prostate cancer in Caucasian men: results from a population-based case-control study. Cancer Causes Control. 2009;20(9):1617-22.

34. Preston MA, et al. Metformin use and prostate cancer risk. Eur Urol. 2014;66(6):1012-20. Epidemiological study showing metformin users were at decreased risk of prostate cancer diagnosis compared to never-users

35. Kordes S, et al. Metformin in patients with advanced pancreatic cancer: a double-blind, randomised, placebo-controlled phase 2 trial. Lancet Oncol. 2015;16(7):839-47.

36. Sayed R, et al. Metformin addition to chemotherapy in stage IV non-small cell lung cancer: an open label randomized controlled study. Asian Pac J Cancer Prev. 2015;16(15):6621-6.

37. Reni M, et al. (Ir)relevance of metformin treatment in patients with metastatic pancreatic cancer: an open-label, randomized phase II trial. Clin Cancer Res. 2016;22(5):1076-85.

38. Higurashi $\mathrm{T}$, et al. Metformin for chemoprevention of metachronous colorectal adenoma or polyps in post-polypectomy patients without diabetes: a multicentre double-blind, placebo-controlled, randomised phase 3 trial. Lancet Oncol. 2016;17(4):47583.

39. Kintzel PE, et al. Increased risk of metabolic syndrome, diabetes mellitus, and cardiovascular disease in men receiving androgen deprivation therapy for prostate cancer. Pharmacotherapy. 2008;28(12):1511-22.

40. Nguyen PL, et al. Adverse effects of androgen deprivation therapy and strategies to mitigate them. Eur Urol. 2015;67(5):825-36.

41. Brown BW, Brauner C, Minnotte MC. Noncancer deaths in white adult cancer patients. J Natl Cancer Inst. 1993;85(12):979-87.

42. Smith MR, Lee H, Nathan DM. Insulin sensitivity during combined androgen blockade for prostate cancer. J Clin Endocrinol Metab. 2006;91(4):1305-8.

43. Lubik AA, et al. Insulin increases de novo steroidogenesis in prostate cancer cells. Cancer Res. 2011;71(17):5754-64.

44. Flanagan J, et al. Presence of the metabolic syndrome is associated with shorter time to castration-resistant prostate cancer. Ann Oncol. 2011;22(4):801-7.

45.• Nobes JP, et al. A prospective, randomized pilot study evaluating the effects of metformin and lifestyle intervention on patients with prostate cancer receiving androgen deprivation therapy. BJU Int. 2012;109(10):1495-502. Randomised trial showing potential benefits of metformin in ADT-treated men
46. Buzzai M, et al. Systemic treatment with the antidiabetic drug metformin selectively impairs p53-deficient tumor cell growth. Cancer Res. 2007;67(14):6745-52.

47. Cantrell LA, et al. Metformin is a potent inhibitor of endometrial cancer cell proliferation - implications for a novel treatment strategy. Gynecol Oncol. 2010;116(1):92-8.

48. Gotlieb $\mathrm{WH}$, et al. In vitro metformin anti-neoplastic activity in epithelial ovarian cancer. Gynecol Oncol. 2008;110(2):246-50.

49. Demir U, et al. Metformin anti-tumor effect via disruption of the MID1 translational regulator complex and AR downregulation in prostate cancer cells. BMC Cancer. 2014;14:52.

50. Liu B, et al. Metformin induces unique biological and molecular responses in triple negative breast cancer cells. Cell Cycle. 2009;8(13):2031-40.

51. Mitsuhashi A, et al. Effects of metformin on endometrial cancer cell growth in vivo: a preoperative prospective trial. Cancer. 2014;120(19):2986-95.

52. Hadad SM, et al. Evidence for biological effects of metformin in operable breast cancer: biomarker analysis in a pre-operative window of opportunity randomized trial. Breast Cancer Res Treat. 2015;150(1):149-55.

53. Niraula $\mathrm{S}$, et al. Metformin in early breast cancer: a prospective window of opportunity neoadjuvant study. Breast Cancer Res Treat. 2012;135(3):821-30.

$54 . \bullet$ Joshua AM, et al. A pilot 'window of opportunity' neoadjuvant study of metformin in localised prostate cancer. Prostate Cancer Prostatic Dis. 2014;17(3):252-8. Demonstrates immunohistochemical changes in patient's prostate samples after antidiabetic doses of metformin

55. Sivalingam VN, et al. Measuring the biological effect of presurgical metformin treatment in endometrial cancer. Br J Cancer. 2016;114(3):281-9.

56. Behnsawy HM, et al. Expression patterns of epithelialmesenchymal transition markers in localized prostate cancer: significance in clinicopathological outcomes following radical prostatectomy. BJU Int. 2013;111(1):30-7.

57. $\mathrm{Qu} \mathrm{C}$, et al. Metformin reverses multidrug resistance and epithelialmesenchymal transition (EMT) via activating AMP-activated protein kinase (AMPK) in human breast cancer cells. Mol Cell Biochem. 2014;386(1-2):63-71.

58. Cerezo M, et al. Metformin blocks melanoma invasion and metastasis development in AMPK/p53-dependent manner. Mol Cancer Ther. 2013;12(8):1605-15.

59. Chou CC, et al. AMPK reverses the mesenchymal phenotype of cancer cells by targeting the Akt-MDM2-Foxo3a signaling axis. Cancer Res. 2014;74(17):4783-95.

60. Lee YS, et al. AMP kinase acts as a negative regulator of RANKL in the differentiation of osteoclasts. Bone. 2010;47(5):926-37.

61. Mai QG, et al. Metformin stimulates osteoprotegerin and reduces RANKL expression in osteoblasts and ovariectomized rats. J Cell Biochem. 2011;112(10):2902-9.

62. Gao Y, et al. Effect of the anti-diabetic drug metformin on bone mass in ovariectomized rats. Eur J Pharmacol. 2010;635(1-3):231-6.

63. Culig Z, Santer FR. Androgen receptor signaling in prostate cancer. Cancer Metastasis Rev. 2014;33(2-3):413-27.

64. Wang Y, et al. Metformin represses androgen-dependent and androgen-independent prostate cancers by targeting androgen receptor. Prostate. 2015;75(11):1187-96.

65. Visvader JE, Lindeman GJ. Cancer stem cells in solid tumours: accumulating evidence and unresolved questions. Nat Rev Cancer. 2008;8(10):755-68.

66. Colombel M, et al. Increased expression of putative cancer stem cell markers in primary prostate cancer is associated with progression of bone metastases. Prostate. 2012;72(7):713-20.

67. Seiler D, et al. Enrichment of putative prostate cancer stem cells after androgen deprivation: upregulation of pluripotency 
transactivators concurs with resistance to androgen deprivation in LNCaP cell lines. Prostate. 2013;73(13):1378-90.

68. Hirsch HA, et al. Metformin selectively targets cancer stem cells, and acts together with chemotherapy to block tumor growth and prolong remission. Cancer Res. 2009;69(19):7507-11.

69. Lonardo E, et al. Metformin targets the metabolic Achilles heel of human pancreatic cancer stem cells. PLoS One. 2013;8(10): e76518.

70. Iliopoulos D, Hirsch HA, Struhl K. Metformin decreases the dose of chemotherapy for prolonging tumor remission in mouse xenografts involving multiple cancer cell types. Cancer Res. 2011;71(9): 3196-201.

71. Babcook MA, et al. Synergistic simvastatin and metformin combination chemotherapy for osseous metastatic castration-resistant prostate cancer. Mol Cancer Ther. 2014;13(10):2288-302.
72. Yue W, et al. Metformin combined with aspirin significantly inhibit pancreatic cancer cell growth in vitro and in vivo by suppressing anti-apoptotic proteins Mcl-1 and Bcl-2. Oncotarget. 2015;6(25): 21208-24.

73. Talarico G, et al. Aspirin and atenolol enhance metformin activity against breast cancer by targeting both neoplastic and microenvironment cells. Sci Rep. 2016;6:18673.

74. Colquhoun AJ, et al. Metformin enhances the antiproliferative and apoptotic effect of bicalutamide in prostate cancer. Prostate Cancer Prostatic Dis. 2012;15(4):346-52.

75. Zhang T, et al. Metformin sensitizes prostate cancer cells to radiation through EGFR/p-DNA-PKCS in vitro and in vivo. Radiat Res. 2014;181(6):641-9.

76. Zannella VE, et al. Reprogramming metabolism with metformin improves tumor oxygenation and radiotherapy response. Clin Cancer Res. 2013;19(24):6741-50. 